\title{
Matrix Formulation of the Cauer Ladder Network Method for Efficient Eddy-Current Analysis
}

$\operatorname{AUTHOR}(\mathrm{S})$ :

Matsuo, Tetsuji; Kameari, Akihisa; Sugahara, Kengo; Shindo, Yuji

\section{CITATION:}

Matsuo, Tetsuji ...[et al]. Matrix Formulation of the Cauer Ladder Network Method for Efficient Eddy-Current Analysis. IEEE Transactions on Magnetics 2018, 54(11): 7205805.

\section{ISSUE DATE:}

2018-11

URL:

http://hdl.handle.net/2433/243800

\section{RIGHT:}

(c) 2018 IEEE. Personal use of this material is permitted. Permission from IEEE must be obtained for all other uses, in any current or future media, including reprinting/republishing this material for advertising or promotional purposes,

creating new collective works, for resale or redistribution to servers or lists, or reuse of any copyrighted component of this work in other works.; This is not the published version. Please cite only the published version.; この論文は出版社版

でありません。引用の際には出版社版をご確認ご利用ください。 


\title{
Matrix Formulation of the Cauer Ladder Network Method for Efficient Eddy-current Analysis
}

\author{
Tetsuji Matsuo $^{1}$, Akihisa Kameari ${ }^{2}$, Kengo Sugahara ${ }^{3}$, and Yuji Shindo ${ }^{4}$ \\ ${ }^{1}$ Graduate School of Engineering, Kyoto University, Kyoto 615-8510, Japan \\ ${ }^{2}$ Science Solutions International Laboratory, Inc., Tokyo 153-0065, Japan \\ ${ }^{3}$ Faculty of Science and Engineering, Kindai University, Higashiosaka 577-8502, Japan \\ ${ }^{4}$ Kawasaki Heavy Industries, Ltd., Akashi 673-8666, Japan
}

\begin{abstract}
A matrix formulation of the Cauer ladder network (CLN) method is derived using the finite element method to clarify the mathematical aspects of the CLN method. The CLN method directly yields orthogonal expansions of electric and magnetic fields that ensure the equivalence of the Cauer network to the eddy-current field. The CLN method is as exact as the Padé approximation via Lanczos (PVL) process but more efficiently provides the circuit parameters and the orthogonal expansion than the PVL.
\end{abstract}

Index Terms - Cauer circuit, eddy current, finite element method, model order reduction

\section{INTRODUCTION}

A DVANCED power control often requires detailed eddycurrent analyses of electric machines handling thin skin depth due to high-frequency switching. The Cauer circuit [1][2] is an efficient and exact representation of the eddycurrent field in magnetic sheets and cylinders for a wide frequency range, where magnetic and electric fields are optimally expanded using orthogonal polynomials.

The Cauer circuit representation was recently extended to describe general eddy-current fields powered by the finite element (FE) method. This method is called the Cauer ladder network (CLN) method [3][4] and retains a clear physical meaning based on the orthogonal function expansion. The generality of the CLN method is similar to that of model order reduction (MOR) methods; e.g., the Padé approximation via the Lanczos (PVL) process [5][6]. The relation between the CLN method and other model order reduction methods has not yet been clarified because of the physics-based derivation of the CLN method.

This paper derives a matrix formulation of the CLN method to clarify mathematical aspects of the CLN method. The orthogonality of function expansion is first derived to prove the equivalence of the Cauer network and eddy-current field. The relation with the Lanczos process and a comparison with the PVL method are then presented.

\section{MATRIX-BASED FORMULATION OF THE CLN METHOD}

\section{A. Finite Element Form of the CLN Method}

Using the edge element $\boldsymbol{w}_{i}{ }_{i}$ and facial element $\boldsymbol{w}_{i}{ }_{i}$ [7], the vector potential $\boldsymbol{A}$, electric field $\boldsymbol{E}$ and magnetic flux density $\boldsymbol{B}$ are represented in FE space as

$$
\boldsymbol{A}=\sum_{i} a_{i} \boldsymbol{w}_{i}^{1}, \quad \boldsymbol{E}=\sum_{i} e_{i} \boldsymbol{w}_{i}^{1}, \quad \boldsymbol{B}=\sum_{j} b_{j} \boldsymbol{w}_{j}^{2}=\operatorname{curl} \boldsymbol{A}
$$

where $a_{i}$ and $e_{i}$ are the line integrals of $\boldsymbol{A}$ and $\boldsymbol{E}$ on edge $i$, and $b_{j}$ is the surface integral of $\boldsymbol{B}$ on face $j$. Variable vectors are defined as

$\boldsymbol{a}=\left[a_{1}, a_{2}, \ldots\right]^{\mathrm{T}}, \boldsymbol{e}=\left[e_{1}, e_{2}, \ldots\right]^{\mathrm{T}}, \boldsymbol{b}=\left[b_{1}, b_{2}, \ldots\right]^{\mathrm{T}}$

which satisfies

$\boldsymbol{b}=\boldsymbol{C a}$

where $\boldsymbol{C}$ is the edge-face incident matrix [7]. The reluctivity matrix and conductivity matrix are defined as

$$
\begin{aligned}
& \boldsymbol{v}=\left\{v_{i j}\right\}, v_{i j}=\int_{\Omega} \frac{1}{\mu} \boldsymbol{w}_{i}^{2} \cdot \boldsymbol{w}_{j}^{2} \mathrm{~d} \Omega, \\
& \boldsymbol{\sigma}=\left\{\sigma_{i j}\right\}, \sigma_{i j}=\int_{\Omega} \sigma \boldsymbol{w}_{i}^{1} \cdot \boldsymbol{w}_{j}^{1} \mathrm{~d} \Omega
\end{aligned}
$$

where $\Omega$ is the analysis domain, $\mu$ is the permeability and $\sigma$ is the conductivity. The eddy-current field is governed by

$\boldsymbol{C}^{\mathrm{T}} \boldsymbol{v b}=\boldsymbol{K a}=\boldsymbol{\sigma e}+\boldsymbol{j}_{0}, \boldsymbol{C e}=-\mathrm{j} \omega \boldsymbol{b}=-\mathrm{j} \omega \boldsymbol{C a}$

where $\boldsymbol{j}_{0}$ is the discretized source current density and $\boldsymbol{K}$ is the coefficient matrix written as [8]

$$
\boldsymbol{K}=\boldsymbol{C}^{\mathrm{T}} \boldsymbol{v} \boldsymbol{C}
$$

The condition $\boldsymbol{\sigma} \boldsymbol{e}+\boldsymbol{j}_{0} \subseteq \operatorname{Range}(\boldsymbol{K})$ is required to guarantee the existence of the $\boldsymbol{K}^{-1}$ operator. If necessary, a gauge condition is imposed to (5).

The CLN method is described by the recurrence formulae below to generate orthogonal basis vectors as 
$\boldsymbol{K}\left(\boldsymbol{a}_{2 n+1}-\boldsymbol{a}_{2 n-1}\right)=\frac{1}{\lambda_{2 n}} \boldsymbol{\sigma} \boldsymbol{e}_{2 n}$

$\boldsymbol{e}_{2 n+2}-\boldsymbol{e}_{2 n}=-\frac{1}{\lambda_{2 n+1}} \boldsymbol{a}_{2 n+1}$

where $\boldsymbol{a}_{2 n-1}$ and $\boldsymbol{e}_{2 n}$ are orthogonal basis vectors for the vector potential and electric field and

$\lambda_{2 n}=\boldsymbol{e}_{2 n}^{\mathrm{T}} \boldsymbol{\sigma} \boldsymbol{e}_{2 n}, \lambda_{2 n+1}=\boldsymbol{a}_{2 n+1}^{\mathrm{T}} \boldsymbol{K} \boldsymbol{a}_{2 n+1}$.

A unit power source is given to start the CLN procedure. For example, if a unit direct voltage is given as the boundary condition, the initial condition is given with the electrostatic field $\boldsymbol{e}_{0}$ as

$\boldsymbol{a}_{-1}=0, \boldsymbol{e}_{0}=-\boldsymbol{G} \boldsymbol{\varphi}$

where $\boldsymbol{\varphi}$ is the variable vector representing scaler potential and $\boldsymbol{G}$ is the node-edge incidence matrix corresponding to the grad operator, which satisfies $\boldsymbol{C G}=0$. If a unit direct current is given as the power input and it imposes current density $\boldsymbol{j}_{0}$, the initial condition is given as

$\boldsymbol{e}_{0}=0, \boldsymbol{a}_{1}=\boldsymbol{K}^{-1} \boldsymbol{j}_{0}$

For the convenience of discussion, the latter condition is included in the former condition by setting

$\boldsymbol{a}_{-1}=0, \boldsymbol{\sigma} \boldsymbol{e}_{0}=\boldsymbol{j}_{0}$

\section{B. Derivation of orthogonality}

The orthogonality of basis vectors is derived by induction as follows. The multiplication of (7) and (8) gives

$-\frac{1}{\lambda_{2 k-1}} \boldsymbol{a}_{2 k-1}^{\mathrm{T}} \boldsymbol{K}\left(\boldsymbol{a}_{2 n+1}-\boldsymbol{a}_{2 n-1}\right)=\frac{1}{\lambda_{2 n}}\left(\boldsymbol{e}_{2 k}-\boldsymbol{e}_{2 k-2}\right)^{\mathrm{T}} \boldsymbol{\sigma} \boldsymbol{e}_{2 n}$.

From (9), (10), and (13) with $n=0$ and $k=1$, it holds that

$\boldsymbol{a}_{-1}^{\mathrm{T}} \boldsymbol{K} \boldsymbol{a}_{1}=0, \boldsymbol{e}_{2}^{\mathrm{T}} \boldsymbol{\sigma} \boldsymbol{e}_{0}=0$.

It is supposed that

$\boldsymbol{a}_{2 i-1}^{\mathrm{T}} \boldsymbol{K} \boldsymbol{a}_{2 j-1}=0, \boldsymbol{e}_{2 i}^{\mathrm{T}} \boldsymbol{\sigma} \boldsymbol{e}_{2 j}=0 \quad(0 \leq i<j \leq n)$.

From (9), (13) and (15),

$\boldsymbol{a}_{2 k-1}^{\mathrm{T}} \boldsymbol{K} \boldsymbol{a}_{2 n+1}=0 \quad(k \leq n)$

is obtained. By replacing $(k, n)$ in $(13)$ with $(n+1, k)$, it holds that $-\frac{1}{\lambda_{2 n+1}} \boldsymbol{a}_{2 n+1}^{\mathrm{T}} \boldsymbol{K}\left(\boldsymbol{a}_{2 k+1}-\boldsymbol{a}_{2 k-1}\right)=\frac{1}{\lambda_{2 k}}\left(\boldsymbol{e}_{2 n+2}-\boldsymbol{e}_{2 n}\right)^{\mathrm{T}} \boldsymbol{\sigma} \boldsymbol{e}_{2 k}$.

From (9), (15) and (17),

$\boldsymbol{e}_{2 k}^{\mathrm{T}} \boldsymbol{\sigma} \boldsymbol{e}_{2 n+2}=0 \quad(k \leq n)$

is obtained. The orthogonality below is thus proven by induction:

$\boldsymbol{e}_{2 i}^{\mathrm{T}} \boldsymbol{\sigma} \boldsymbol{e}_{2 j}=\delta_{i j} \lambda_{2 i}, \quad \boldsymbol{a}_{2 i+1}^{\mathrm{T}} \boldsymbol{K} \boldsymbol{a}_{2 j+1}=\delta_{i j} \lambda_{2 i+1}$

where $\delta_{i j}$ is Kronecker's delta.

\section{Derivation of circuit equations}

Electromagnetic fields are expanded as

$\boldsymbol{a}=\sum_{n} I_{2 n+1} \boldsymbol{a}_{2 n+1}, \quad \boldsymbol{e}=\sum_{n} V_{2 n} \boldsymbol{e}_{2 n}$

The substitution of (20) into (5) gives

$\boldsymbol{K} \sum_{n} I_{2 n+1} \boldsymbol{a}_{2 n+1}=\boldsymbol{\sigma} \sum_{n} V_{2 n} \boldsymbol{e}_{2 n}$

$C \sum_{n} V_{2 n} \boldsymbol{e}_{2 n}=-\mathrm{j} \omega \sum_{n} I_{2 n+1} \boldsymbol{C} \boldsymbol{a}_{2 n+1}$.

From (22), the electric field is given as

$\sum_{n} V_{2 n} \boldsymbol{e}_{2 n}=-\mathrm{j} \omega \sum_{n} I_{2 n+1} \boldsymbol{a}_{2 n+1}-V_{\mathrm{S}} \boldsymbol{G} \boldsymbol{\varphi}$

where $V_{\mathrm{S}}$ is the source voltage. Multiplying (23) by $\left(1 / \lambda_{2 k}\right) \boldsymbol{\sigma} \boldsymbol{e}_{2 k}$ and using (7) give

$$
\begin{aligned}
& \frac{1}{\lambda_{2 k}} \boldsymbol{e}_{2 k}^{\mathrm{T}} \boldsymbol{\sigma}\left(\sum_{n} V_{2 n} \boldsymbol{e}_{2 n}-V_{\mathrm{S}} \boldsymbol{e}_{0}\right) \\
& \quad=-\mathrm{j} \omega\left(\boldsymbol{a}_{2 k+1}-\boldsymbol{a}_{2 k-1}\right)^{\mathrm{T}} \boldsymbol{K} \sum_{n} I_{2 n+1} \boldsymbol{a}_{2 n+1}
\end{aligned}
$$

From (19) and (24), it holds that

$V_{2 k}=-\mathrm{j} \omega\left(\lambda_{2 k+1} I_{2 k+1}-\lambda_{2 k-1} I_{2 k-1}\right) \quad(k=1,2, \ldots)$

$V_{0}=V_{\mathrm{S}}-\mathrm{j} \omega \lambda_{1} I_{1}$

Multiplying (21) by $-\left(1 / \lambda_{2 k+1}\right) \boldsymbol{a}_{2 k+1}$ and using (8) give

$-\frac{1}{\lambda_{2 k+1}} \boldsymbol{a}_{2 k+1}^{\mathrm{T}} \boldsymbol{K} \sum_{n} I_{2 n+1} \boldsymbol{a}_{2 n+1}=\left(\boldsymbol{e}_{2 k+2}-\boldsymbol{e}_{2 k}\right)^{\mathrm{T}} \boldsymbol{\sigma} \sum_{n} V_{2 n} \boldsymbol{e}_{2 n}$.

From (19) and (26), it holds that

$-I_{2 k+1}=\lambda_{2 k+2} V_{2 k+2}-\lambda_{2 k} V_{2 k} \quad(k=0,1, \ldots)$.

Equations (26) and (28) represent the Cauer circuit in Fig. 1, where $R_{2 k}=1 / \lambda_{2 k}$ and $L_{2 k+1}=\lambda_{2 k+1}$. 


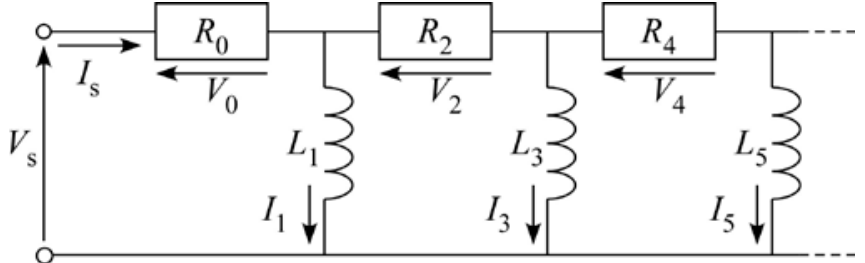

Fig. 1. Cauer circuit.

\section{Derivation of the CLN method from circuit equations}

The substitution of (25) and (27) into (21) and (23) results in

$$
\begin{aligned}
& -\mathrm{j} \omega \sum_{n}\left(\lambda_{2 n+3} I_{2 n+3}-\lambda_{2 n+1} I_{2 n+1}\right) \boldsymbol{e}_{2 n+2}+\left(V_{\mathrm{S}}-\mathrm{j} \omega \lambda_{1} I_{1}\right) \boldsymbol{e}_{0} \\
& \quad=\mathrm{j} \omega \sum_{n} \lambda_{2 n+1} I_{2 n+1}\left(\boldsymbol{e}_{2 n+2}-\boldsymbol{e}_{2 n}\right)+V_{\mathrm{S}} \boldsymbol{e}_{0} \\
& \quad=-\mathrm{j} \omega \sum_{n} I_{2 n+1} \boldsymbol{a}_{2 n+1}-V_{\mathrm{S}} \boldsymbol{G} \boldsymbol{\varphi} \\
& \boldsymbol{K} \sum_{n}\left(\lambda_{2 n} V_{2 n}-\lambda_{2 n+2} V_{2 n+2}\right) \boldsymbol{a}_{2 n+1} \\
& =\boldsymbol{K} \sum_{n} \lambda_{2 n} V_{2 n}\left(\boldsymbol{a}_{2 n+1}-\boldsymbol{a}_{2 n-1}\right)=\boldsymbol{\sigma} \sum_{n} V_{2 n} \boldsymbol{e}_{2 n} .
\end{aligned}
$$

To satisfy (28) and (29) for arbitrary $V_{\mathrm{S}}, V_{2 n}$ and $I_{2 n+1}$, (7) and (8) are required, which confirms the equivalence of the CLN and eddy-current field.

\section{E. Relation with the Lanczos algorithm}

From (7) and (8), three-term recurrence formulae for $\boldsymbol{a}_{2 n-1}$ and $\boldsymbol{e}_{2 n}$ are obtained as

$$
\begin{aligned}
& \lambda_{2 n} \boldsymbol{a}_{2 n+1}+\left[\frac{1}{\lambda_{2 n-1}} \boldsymbol{K}^{-1} \boldsymbol{\sigma}-\left(\lambda_{2 n}+\lambda_{2 n-2}\right) \mathbf{1}\right] \boldsymbol{a}_{2 n-1}+\lambda_{2 n-2} \boldsymbol{a}_{2 n-3}=0 \\
& \lambda_{2 n+2} \boldsymbol{e}_{2 n+2}+\left[\frac{1}{\lambda_{2 n}} \boldsymbol{K}^{-1} \boldsymbol{\sigma}-\left(\lambda_{2 n+1}+\lambda_{2 n-1}\right) \mathbf{1}\right] \boldsymbol{e}_{2 n}+\lambda_{2 n-1} \boldsymbol{e}_{2 n-2}=0
\end{aligned}
$$

where $\mathbf{1}$ is the unit matrix. By setting

$\boldsymbol{e}_{n}^{\prime}=\boldsymbol{e}_{2 n} / \sqrt{\lambda_{2 n}}, \quad \boldsymbol{j}_{n}^{\prime}=\boldsymbol{\sigma} \boldsymbol{e}_{2 n} / \sqrt{\lambda_{2 n}}$,

(31) can be rewritten as a bi-Lanczos process in the form

$\boldsymbol{K}^{-1} \boldsymbol{\sigma} \boldsymbol{e}_{n}^{\prime}=r_{n} \boldsymbol{e}_{n+1}^{\prime}+q_{n} \boldsymbol{e}_{n}^{\prime}+r_{n-1} \boldsymbol{e}_{n-1}^{\prime}$

$\boldsymbol{\sigma} \boldsymbol{K}^{-1} \boldsymbol{j}_{n}^{\prime}=r_{n} \boldsymbol{j}_{n+1}^{\prime}+q_{n} \boldsymbol{j}_{n}^{\prime}+r_{n-1} \boldsymbol{j}_{n-1}^{\prime}$,

where $\boldsymbol{j}_{i}{ }_{i}^{\mathrm{T}} \boldsymbol{e}_{j}{ }_{j}=\delta_{i j}$ and

$$
\begin{aligned}
& q_{n}=\boldsymbol{j}_{n}^{\prime \mathrm{T}} \boldsymbol{K}^{-1} \boldsymbol{\sigma} \boldsymbol{e}_{n}^{\prime}=\lambda_{2 n}\left(\lambda_{2 n+1}+\lambda_{2 n-1}\right) \\
& r_{n}=\boldsymbol{j}_{n+1}^{\prime \mathrm{T}} \boldsymbol{K}^{-1} \boldsymbol{\sigma} \boldsymbol{e}_{n}^{\prime}=\boldsymbol{j}_{n}^{\prime \mathrm{T}} \boldsymbol{K}^{-1} \boldsymbol{\sigma} \boldsymbol{e}_{n+1}^{\prime}=-\lambda_{2 n+1} \sqrt{\lambda_{2 n+2} \lambda_{2 n}} .
\end{aligned}
$$

By setting

$$
\boldsymbol{a}_{n}^{\prime}=\boldsymbol{a}_{2 n-1} / \sqrt{\lambda_{2 n-1}}, \quad \boldsymbol{j}_{n}^{\prime \prime}=\boldsymbol{K} \boldsymbol{a}_{2 n-1} / \sqrt{\lambda_{2 n-1}},
$$

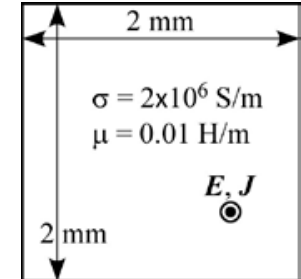

Fig. 2. Square iron bar.

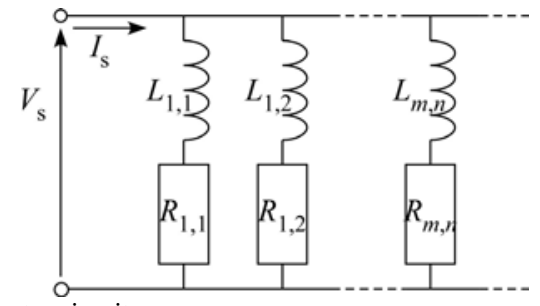

Fig. 3. Foster circuit

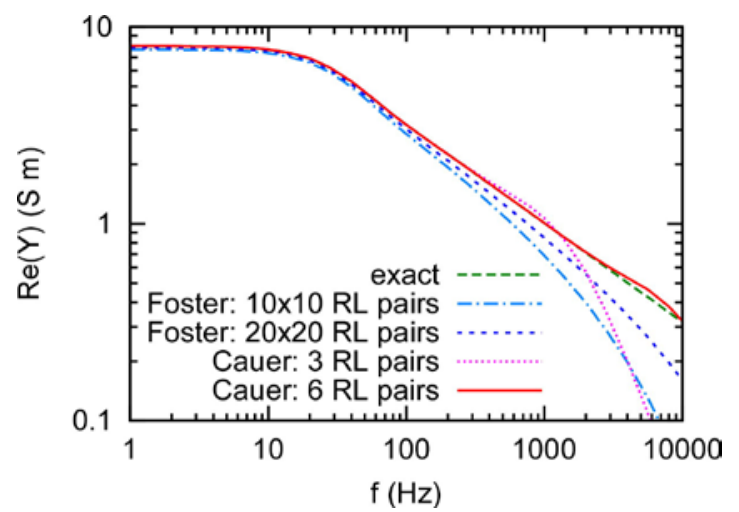

Fig. 4. Frequency dependence of admittance $Y=I_{\mathrm{S}} / V_{\mathrm{S}}$ per unit length.

(30) can be rewritten as another bi-Lanczos process in the form

$\boldsymbol{K}^{-1} \boldsymbol{\sigma} \boldsymbol{a}_{n}^{\prime}=t_{n} \boldsymbol{a}_{n+1}^{\prime}+s_{n} \boldsymbol{a}_{n}^{\prime}+t_{n-1} \boldsymbol{a}_{n-1}^{\prime}$,

$\boldsymbol{\sigma} \boldsymbol{K}^{-1} \boldsymbol{j}_{n}^{\prime \prime}=t_{n} \boldsymbol{j}_{n+1}^{\prime \prime}+s_{n} \boldsymbol{j}_{n}^{\prime \prime}+t_{n-1} \boldsymbol{j}_{n-1}^{\prime \prime}$

where $\boldsymbol{j}_{i}{ }_{i}^{\mathrm{T}} \boldsymbol{a}_{j}{ }_{j}=\delta_{i j}$ and

$s_{n}=\boldsymbol{j}_{n}^{\prime \prime \mathrm{T}} \boldsymbol{K}^{-1} \boldsymbol{\sigma} \boldsymbol{a}_{n}^{\prime}=\lambda_{2 n-1}\left(\lambda_{2 n}+\lambda_{2 n-2}\right)$

$t_{n}=\boldsymbol{j}_{n+1}^{\prime \prime \mathrm{T}} \boldsymbol{K}^{-1} \boldsymbol{\sigma} \boldsymbol{a}_{n}^{\prime}=\boldsymbol{j}_{n}^{\prime \prime \mathrm{T}} \boldsymbol{K}^{-1} \boldsymbol{\sigma} \boldsymbol{a}_{n+1}^{\prime}=-\lambda_{2 n} \sqrt{\lambda_{2 n+1} \lambda_{2 n-1}}$

The CLN method thus contains two bi-Lanczos processes. In contrast, the PVL method consists of one bi-Lanczos process. A performance comparison of the CLN method with the PVL method is made in the following section.

\section{Computational Results}

Two simple but illustrative eddy-current problems are solved to demonstrate the efficiency and accuracy of the CLN method.

\section{A. Square Iron Bar}

An iron bar with square cross-section [Fig. 2] has conductivity of $2 \times 10^{6} \mathrm{~S} / \mathrm{m}$ and permeability of $0.01 \mathrm{H} / \mathrm{m}$. A unit electric field is imposed at the surface of the iron bar. The 
analytical solution is given by Fourier expansion, which is represented by the Foster circuit shown in Fig. 3, where the ( $m$, $n)$-th R-L pair corresponds to the $(2 m-1,2 n-1)$-th sinusoidal basis function. Fig. 4 compares the frequency dependence of admittance per unit length, where the exact solution is given by the Foster circuit with $2000 \times 2000 \mathrm{R}-\mathrm{L}$ pairs. The Cauer circuit with only three or six R-L pairs accurately reconstructs the wide range of the frequency dependence whereas the Foster circuit requires a large number of R-L pairs.

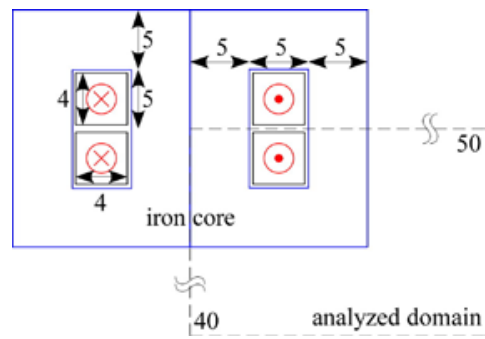

Fig. 5. Iron-cored inductor with dimensions given in millimeters.

\section{B. Iron-cored Inductor}

Fig. 5 illustrates the iron-cored inductor and its analyzed domain. A conductor bar with a square cross-section of $4 \mathrm{~mm}$ $\times 4 \mathrm{~mm}$ has conductivity of $4 \times 10^{7} \mathrm{~S} / \mathrm{m}$ and permeability of $\mu_{0}=4 \pi \times 10^{-7} \mathrm{H} / \mathrm{m}$. A bulk-type iron core has permeability of $1000 \mu_{0}$ and conductivity of $1 \times 10^{6} \mathrm{~S} / \mathrm{m}$. The governing equation of the eddy-current field is

$\operatorname{div}(v \operatorname{grad} \boldsymbol{A})=-\sigma\left(\boldsymbol{E}_{0}-\frac{\partial \boldsymbol{A}}{\partial t}\right)$

where $\boldsymbol{E}_{0}=\left(0,0, E_{0}\right)$ is the imposed electric field in the conductor bar. The coil current is given as

$$
I=\int_{\text {Scoil }} \sigma\left(\boldsymbol{E}_{0}-\frac{\partial \boldsymbol{A}}{\partial t}\right) \cdot \mathrm{d} \boldsymbol{S}
$$

where $\boldsymbol{E}_{0}-\partial \boldsymbol{A} / \partial t$ is the electric field in the conductor bar whose cross-section is $S_{\text {coill }}$. Setting $E_{0}$ to the unit electric field in the conductor, the CLN yields the admittance $Y$ of the inductor of 1/4 part per unit length, which gives the relation

$$
I=Y E_{0} .
$$

Fig. 6 shows the frequency response of the inductor by plotting $\operatorname{Re}(Y)$ and inductance $L=\operatorname{Im}(1 / Y) / \omega$. In the figure, the label ' $n L$ ' means the use of $n$-pairs of $\left(R_{0}, L_{1}\right), \ldots,\left(R_{2 n-2}\right.$, $L_{2 n-1}$ ) terminated by $L_{2 n-1}$ while the label ' $n R$ ' means the use of $n$-pairs of $R-L$ and $R_{2 n}$. Both are $n$-th order CLN approximations that require $n$-times operation of $\boldsymbol{K}^{-1}$. For comparison, the figure also plots the frequency response given by the time-dependent FE eddy-current analysis. The CLN yields the exact response with low-order approximations.

For comparison, this subsection also obtains the admittance using the PVL method [5][6] setting $E_{0}$ and $I$ as the input and output. The computational cost of eddy-current analysis is roughly evaluated by the number of multiplications of $\boldsymbol{K}^{-1}$. The PVL method requires the operation of $\boldsymbol{K}^{-1}$ once in the first step for the initial setting and twice per cycle of the biLanczos procedure, which means the $n$-the order PVL approximation requires $2 n-1$ operations of $\boldsymbol{K}^{-1}$. The number of operations of $\boldsymbol{K}^{-1}$ are indicated in the parentheses in Figs. 6 and 7. Fig. 7 shows that the $n$-th-order PVL method obtains almost the same response given by the $n$-th-order CLN terminated with $R_{2 n}$. The benefits of the CLN method are as follows.

(a) For the $n$-th-order approximation, the CLN method requires $n$ operations of $\boldsymbol{K}^{-1}$ whereas the PVL method requires $2 n-1$ operations.

(b) The CLN method directly yields circuit parameters whereas the PVL method requires an eigen decomposition to obtain the circuit parameters.

(c) The CLN method also directly derives the orthogonal expansion (20).

The magnetic flux distributions due to $\boldsymbol{a}_{1}, \boldsymbol{a}_{3}$ and $\boldsymbol{a}_{5}$ are shown in Fig. 8. Using expansions (20) with these distributions, the CLN method can reconstruct the magnetic and electric fields.

The time-dependent response is next reconstructed using the CLN method for the input of the square waveform of $E_{0}$ shown in Fig. 9(a). Fig. 9(b) plots the current waveform, where the fourth-order CLN gives a good approximation and the eighth-order CLN achieves an exact reconstruction of the waveform. Fig. 10 depicts the magnetic flux lines at $10 \mathrm{~ms}$, where the fourth- and eighth-order CLNs obtain good approximations and the 16-th-order CLN yields exact flux lines.
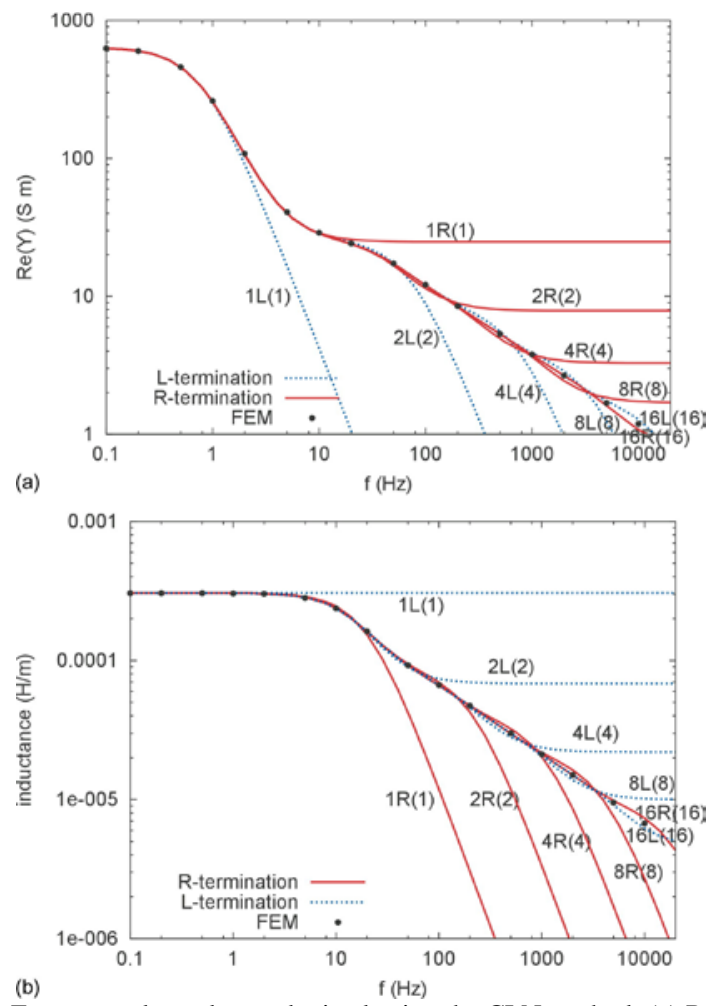

Fig. 6. Frequency dependence obtained using the CLN method: (a) $\operatorname{Re}(Y)$ and (b) inductance. 

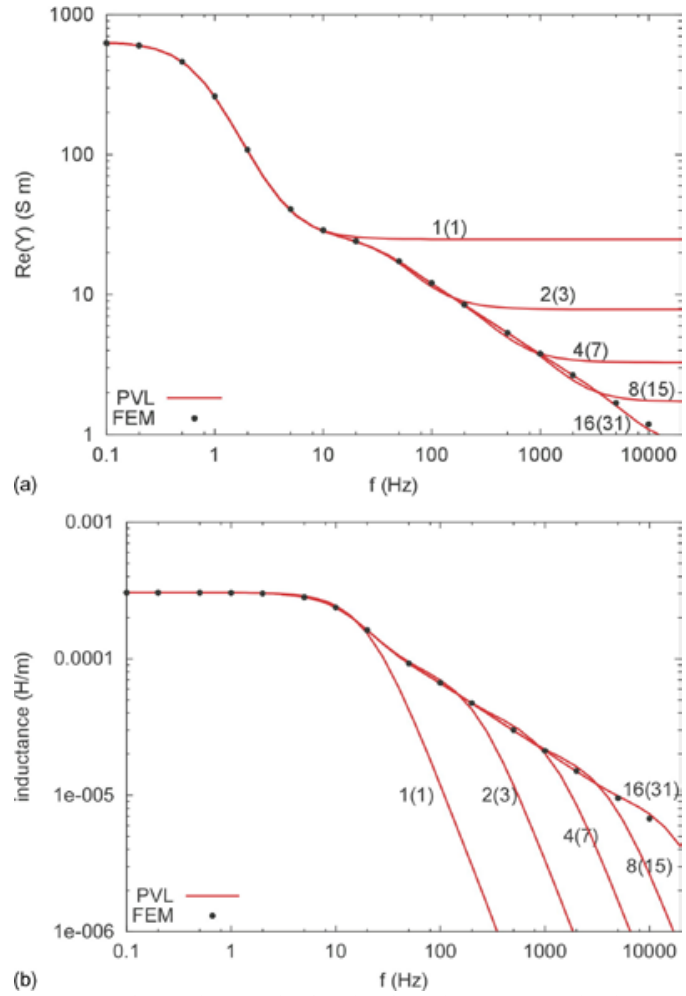

Fig. 7. Frequency dependence obtained using the PVL method: (a) $\operatorname{Re}(Y)$ and (b) inductance.

\section{CONCLUSION}

A matrix formulation of the CLN was derived. Involving two Lanczos processes, the CLN method efficiently yields a circuit representation equivalent to the eddy-current field. The CLN method is as exact as the PVL method and directly provides the circuit parameters and the orthogonal expansion of the eddy-current field with lower computational cost than the PVL method.

\section{ACKNOWLEDGMENT}

T. M. thanks Mr. R. Maeda, M.S. student, Kyoto University, Japan, for his assistance in analyzing the Foster circuit.

\section{REFERENCES}

[1] Y. Shindo, T. Miyazaki, T. Matsuo, "Cauer circuit representation of the homogenized eddy-current field based on the Legendre expansion for a magnetic sheet,” IEEE Trans. Magn., vol. 52, no. 3, 6300504, Mar. 2016.

[2] Y. Shindo, A. Kameari, T. Matsuo, "Efficient circuit representation of eddy-current fields,” COMPEL, vol. 36, Iss: 5, pp. 1457-1473, 2017.

[3] A. Kameari, H. Ebrahimi, K. Sugahara, Y. Shindo, and T. Matsuo, "Cauer ladder network representation of eddy-current fields for model order reduction using finite element method,” IEEE Trans. Magn., vol. 54,7201804, Mar. 2018.

[4] K. Sugahara, A. Kameari, H. Ebrahimi, Y. Shindo and T. Matsuo, "Finite element analysis of unbounded eddy-current problems with Cauer ladder network method,” IEEE Trans. Magn, vol. 54, 7200704, Mar. 2018.

[5] P. Feldmann and R. W. Freund, "Efficient linear circuit analysis by Padé approximation via the Lanczos process," IEEE Trans. Comput.-Aided Design Integr. Circuits Syst., vol. 14, no. 5, pp. 639-649, May 1995.

[6] Y. Sato, and H. Igarashi, "Generation of equivalent circuit from finiteelement model using model order reduction," IEEE Trans. Magn., vol. 52, no. 3, 1100304, Mar. 2016.

[7] A. Bossavit, Computational Electromagnetism, Academic Press, 1998.
[8] T. Tarhasaari, L. Kettunen, A. Bossavit, Some realizations of a discrete Hodge operator: a reinterpretation of finite element techniques, IEEE Trans. Magn., vol. 35, pp. 1494-1497, 1999.

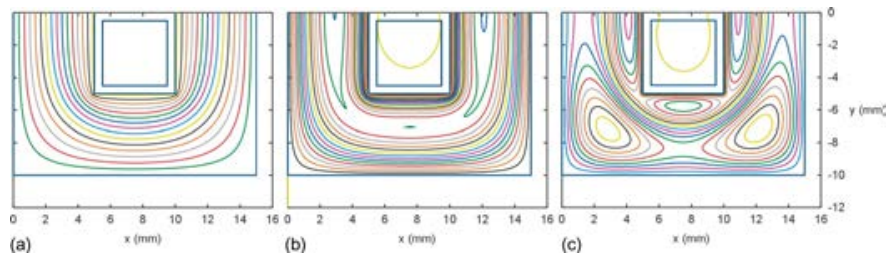

Fig. 8 Magnetic flux lines corresponding to (a) $\boldsymbol{a}_{1}$, (b) $\boldsymbol{a}_{3}$, and (c) $\boldsymbol{a}_{5}$.

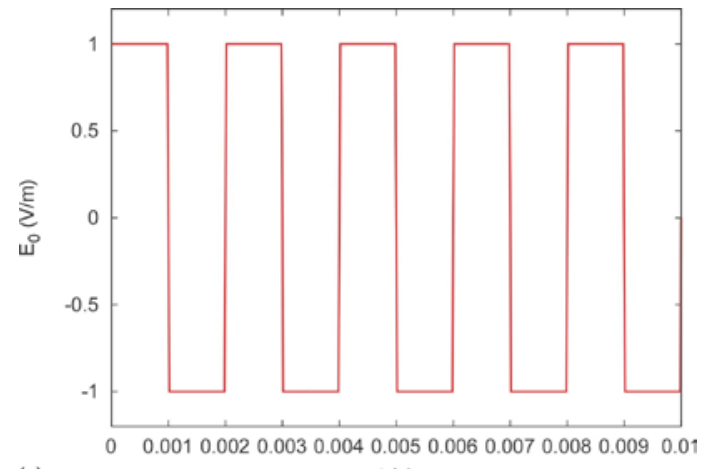

(a) $\mathrm{t}(\mathrm{s})$

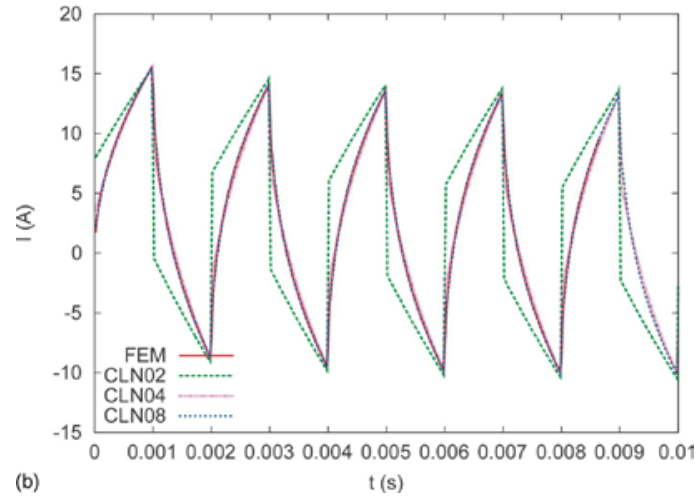

Fig. 9. Time-dependent response: (a) imposed electric field and (b) current.
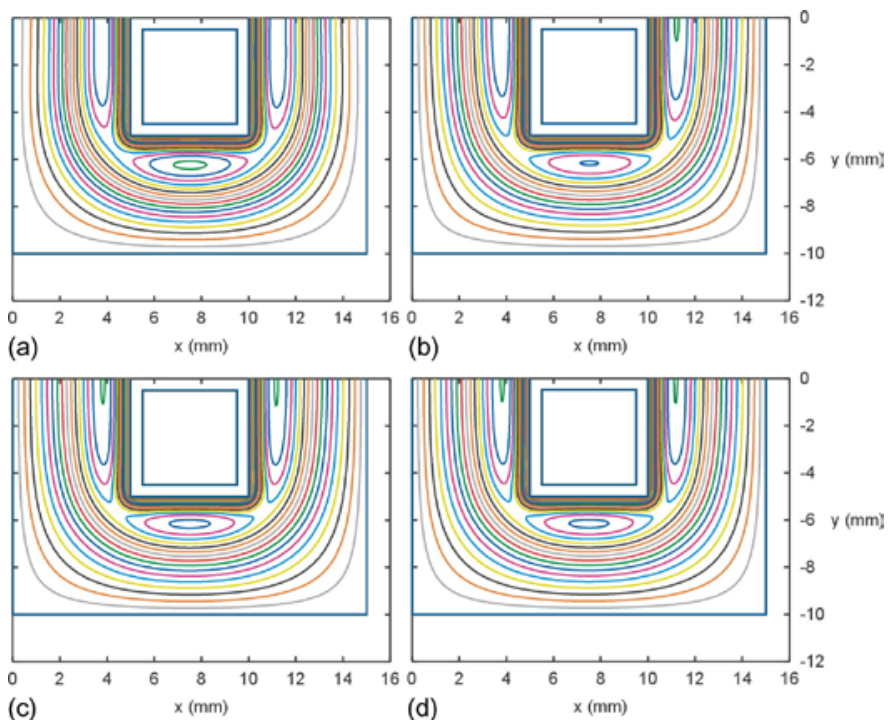

Fig. 10. Magnetic flux lines given by (a) fourth-, (b) eighth-, and (c) 16thorder CLNs and (d) time-dependent FE analysis. 